\title{
Proteomic Signature of Arabidopsis Cell Cultures Exposed to Magnetically Induced Hyper- and Microgravity Environments
}

\author{
Raul Herranz,, ${ }^{1, *}$ Ana I. Manzano,, ${ }^{1, *}$ Jack J.W.A. van Loon, ${ }^{2}$ \\ Peter C.M. Christianen, ${ }^{3}$ and F. Javier Medina ${ }^{1}$
}

\begin{abstract}
Earth-based microgravity simulation techniques are required due to space research constraints. Using diamagnetic levitation, we exposed Arabidopsis thaliana in vitro callus cultures to environments with different levels of effective gravity and magnetic field strengths $(B)$ simultaneously. The environments included simulated $0 g^{*}$ at $B=10.1 \mathrm{~T}$, an internal $1 g^{*}$ control $(B=16.5 \mathrm{~T})$, and hypergravity $\left(2 g^{*}\right.$ at $\left.B=10.1 \mathrm{~T}\right)$. Furthermore, samples were also exposed to altered gravity environments that were created with mechanical devices, such as the Random Positioning Machine (simulated $\mu g$ ) and the Large Diameter Centrifuge $(2 g)$. We have determined the proteomic signature of cell cultures exposed to these altered-gravity environments by means of the difference gel electrophoresis (DiGE) technique, and we have compared the results with microarray-based transcriptomes from the same samples. The magnetic field itself produced a low number of proteomic alterations, but the combination of gravitational alteration and magnetic field exposure produced synergistic effects on the proteome of plants (the number of significant changes is 3-7 times greater). Tandem mass spectrometry identification of 19 overlapping spots in the different conditions corroborates a major role of abiotic stress and secondary metabolism proteins in the molecular adaptation of plants to unusual environments, including microgravity. Key Words: DiGEMicrogravity simulation-Magnetic levitation-Proteome/transcriptome comparison-Callus cell cultures. Astrobiology 13, 217-224.
\end{abstract}

$\mathbf{T}$ O STUDY THE EFFECT of an environment with suppressed gravitational forces, we have to place samples in orbit by means of spaceflights or sounding rockets, or use simulation facilities on the ground. Mechanical facilities for microgravity, such as 2-D clinostats or random positioning machines, and centrifuges for hypergravity, like the Large Diameter Centrifuge (LDC) (Hoson et al., 1992; Kraft et al., 2000; van Loon et al., 2004; van Loon, 2007), are used as experimental approaches to the study of the effects of altered gravity, including gravitation-dependent proteomic analyses (Wang et al., 2006; Barjaktarovic et al., 2007, 2009), and help avoid the costs and constraints of space experimentation. Nevertheless, it is not clear whether the use of such machines creates either a stimulus-free environment with respect to gravity (simulated weightlessness) or an omnilateral gravistimulation with strong mechanical disturbances. An alternative approach to study the response of organisms to changes in gravity is the use of diamagnetic levitation (Beaugnon and Tournier 1991a, 1991b; Berry and Geim 1997; Valles et al., 1997). Since diamagnetic material is repelled by magnetic fields, when a diamagnetic object is positioned in a magnetic field gradient it experiences a magnetic force away from regions of high field. The magnitude of the force is proportional to the product of the field strength $(B)$ by the field gradient (the spatial derivative of the field, $\left.B^{\prime}\right)$. When $\left[B \times B^{\prime}\right]$ is strong enough, this magnetic force can be used to counterbalance the gravitational force, leading to the levitation of a large variety of materials such as water and other fluids. Since the bulk of living organisms is composed of diamagnetic material, mostly water, organisms can be magnetically levitated, provided $\left[B \times B^{\prime}\right]$ is about $1400 \mathrm{~T}^{2} / \mathrm{m}$. Stable magnetic levitation is only possible in a dozen magnets around the world, at a field

\footnotetext{
${ }^{1}$ Centro de Investigaciones Biológicas (CSIC), Madrid, Spain.

${ }^{2}$ Dutch Experiment Support Center, DESC at OCB-ACTA, Free University and University of Amsterdam, Department of Oral Cell Biology, Research Institute MOVE, Amsterdam, the Netherlands.

${ }^{3}$ High Field Magnet Laboratory (HFML), Institute for Molecules and Materials, Radboud University, Nijmegen, the Netherlands.

*These authors have contributed equally to this work.
} 


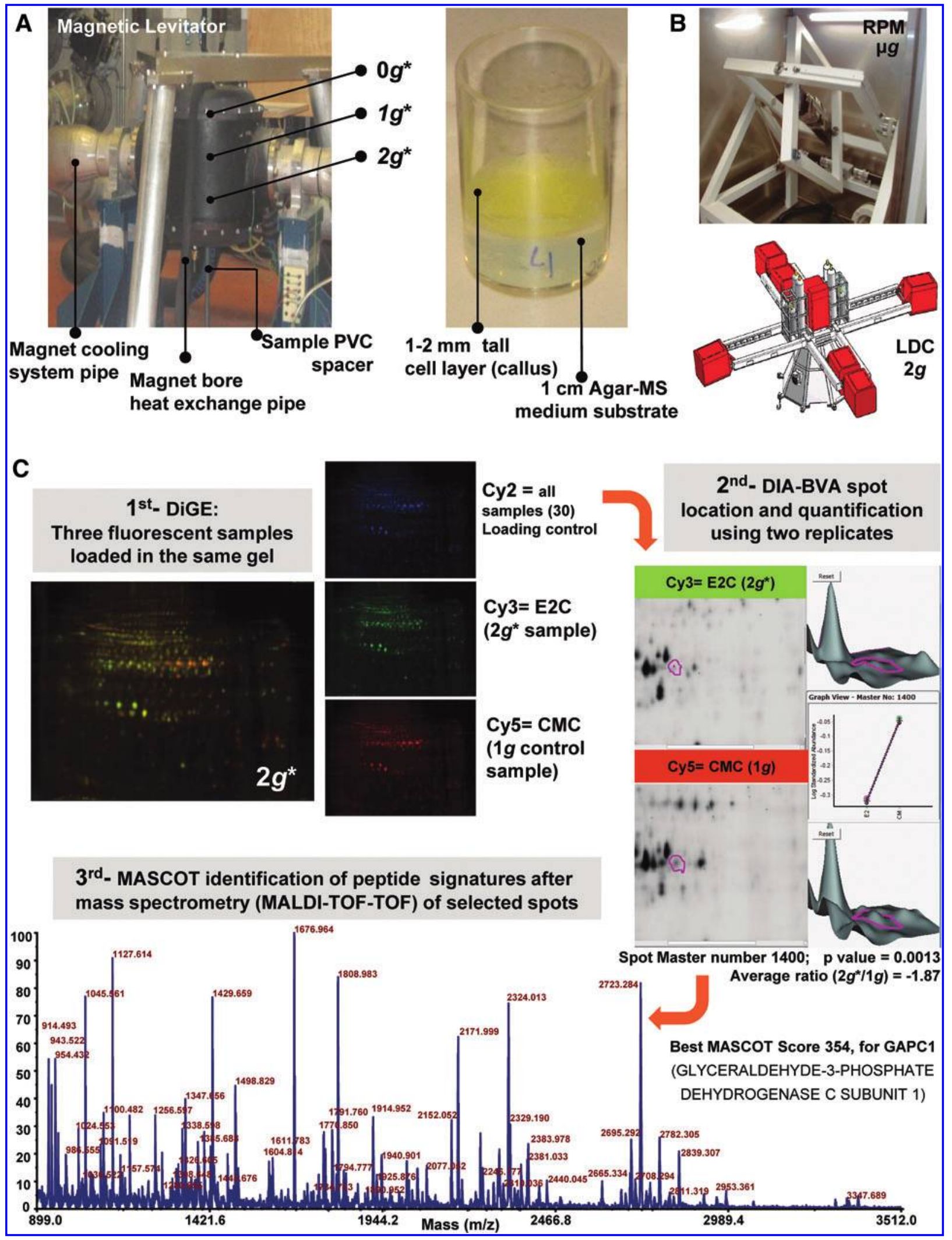

FIG. 1. Altered-gravity ground-based facilities and experimental approach. (A) Magnetic levitator picture including a detail of the sample container and location of the samples in the magnet bore. (B) Mechanical ground-based facilities used to produce altered gravity on ground (RPM as a microgravity simulator and LDC, a $2 g$ centrifuge). (C) Example of the threestep experimental approach performed to locate, quantify, and identify one protein affected by hypergravity treatments (GAPC1). (Color images available online at www.liebertonline.com/ast) 
strength of at least $16 \mathrm{~T}$, so this technology was made available to the space biologist community only some years ago (Valles et al., 2005; Guevorkian and Valles 2006; Beuls et al., 2009; Hammer et al., 2009; Dijkstra et al., 2011; Herranz et al., 2012; Hill et al., 2012; Manzano et al., 2012).

We have studied the effect of an exposure of $200 \mathrm{~min}$ to an environment of altered gravitational and magnetic forces on the overall proteomic profile of Arabidopsis thaliana semisolid cell cultures (callus). The present study is the first systematic multifacility, high-throughput, environmentally controlled collection of experiments that has been performed with the same setup, almost simultaneously in two mechanical facilities (RPM for simulated $\mu g$ and LDC for $2 g$ ) and in a magnet-based facility, with the use of three different effective gravity $\left(g^{*}\right)$ conditions $\left[2 g^{*}, 1 g^{*}\right.$, and $0 g^{*}$, namely, the calculated effective gravity for pure water in the center of each culture chamber, assuming that variations due to differential magnetic susceptibly of intracellular material are below biological sensitive (Schenck 1992; Valles et al., 1997); see also Herranz et al. (2013) for a discussion about terminology]. This made possible inter-experiment comparisons of the results and a pooled analysis with multiple inner controls at similar magnetic fields. Apart from testing what kind of facility would be most suitable and reliable as an altered gravity simulator, a major achievement of this study was the comparison of the proteomic profiles with the transcriptomic results obtained from the same samples [Agilent two-color 44k whole genome microarray data sets, GEO ID: GSE29787 (Manzano et al., 2012)].

A detailed description of the experimental setup, the levitation magnet (Fig. 1A), and the mechanical simulators (Fig. 1B) was provided in a parallel transcriptomic paper (Manzano et al., 2012). In short, we exposed samples to three different conditions ( $g^{*}$ and $B$ fields) within a high field magnet (HFML, Radboud University Nijmegen, the Netherlands) (Perenboom et al., 2004; Wiegers et al., 2010). The magnitude $g^{*}$ denotes the value of the effective gravity (the asterisk reflects the presence of the background magnetic field) in each of the three positions within the magnet (Table 1). In the center of the magnet bore ( $1 g^{*}$ position), the magnetic field strength is maximal (16.5 T), but the magnetic field gradient is zero. At a distance of $81.6 \mathrm{~mm}$ above this position, at $B=10.1 \mathrm{~T}$, the diamagnetic force on water counterbalances the force of gravity, which leads to stable levitation; this is the $0 g^{*}$ position

Table 1. Number of Spots Showing Altered Concentrations $(p<0.05)$ Under DifFERENT EFFective Gravity $\left(g^{*}\right)$ and Magnetic/Mechanical Conditions

\begin{tabular}{|c|c|c|c|c|c|}
\hline $\begin{array}{l}\text { Effective } \\
\text { force }(\mathrm{g})\end{array}$ & $0 g^{*}$ & $1 g^{*}$ & $2 g^{*}$ & $\begin{array}{c}\mu \mathrm{g} \\
R P M\end{array}$ & $\begin{array}{c}2 \mathrm{~g} \\
L D C\end{array}$ \\
\hline Magnetic field $(B)$ & $10.1 \mathrm{~T}$ & $16.5 \mathrm{~T}$ & $10.1 \mathrm{~T}$ & - & - \\
\hline Mechanical forces & - & - & - & YES & YES \\
\hline Spots $\geq 1.2$-fold & $22(13)$ & $4(15)$ & $56(34)$ & $1(0)$ & $1(0)$ \\
\hline Spots $\leq-1.2$-fold & $38(17)$ & $14(20)$ & $61(55)$ & $0(0)$ & $2(0)$ \\
\hline TOTAL & $60(30)$ & $18(35)$ & $117(89)$ & $1(0)$ & $3(0)$ \\
\hline
\end{tabular}

— indicated that magnetic/mechanical perturbations are minimal and similar to the control for those samples. Number of significantly altered spots was determined by using a Student $t$ test $(p<0.05)$ with two replicates. The numbers obtained, including a more variable third replicate, are shown between brackets for comparison. (zero gravity is only reached in a single point). At a distance of $81.6 \mathrm{~mm}$ below the field center, also at $B=10.1 \mathrm{~T}$, the sum of the levitation and gravitational forces produces a $2 g^{*}$ effective force. In both cases, we consider that the effective gravity applied on Arabidopsis culture cells (free of starch statoliths) is similar to the one of water. We base this assumption on the fact that cells' main component is water, and most organic compounds within the cell have similar diamagnetic properties as those of water (Schenck 1992; Valles et al., 1997). Additionally, we exposed samples to a mechanical simulator of microgravity, the Random Positioning Machine ( $\mu g-\mathrm{RPM}$, real random mode), and to the Large Diameter Centrifuge ( $2 g$-LDC) for mechanical generation of hypergravity, both facilities located in the European Space Research and Technology Centre [ESA-ESTEC, Noordwijk, the Netherlands, Fig. 1B (van Loon, 2007; van Loon et al., 2008)]. In each facility, samples were exposed for $200 \mathrm{~min}$, at $22^{\circ} \mathrm{C} \pm 0.1^{\circ} \mathrm{C}$, in darkness. External controls were kept at room temperature $\left(22^{\circ} \mathrm{C} \pm 0.1^{\circ} \mathrm{C}, 1 \mathrm{~g}\right.$ ground gravity), away from the magnet, LDC, or RPM. As a model system, we used callus semisolid cultures of Arabidopsis thaliana prepared from MM2d suspension cultures (Menges and Murray, 2006). For all devices and conditions, the suspension cultures were strewn on the agar surface 1 week before the start of the experiment and grown at $22^{\circ} \mathrm{C}$ to allow the callus to reach maximum density in a $1-2 \mathrm{~mm}$ thick layer to minimize variations in the magnetic field and of the effective $g$-force. The samples were preserved immediately after treatment (less than $2 \mathrm{~min}$ ) by quick freezing in liquid nitrogen and subsequent storage, first in dry ice for transport and then under deep freezing.

Samples were analyzed as to their proteomic profile as summarized in the example in Fig. 1C by the two-dimensional difference gel electrophoresis (2-D-DiGE) technique. Protein was extracted from three biological replicates as described by Barjaktarovic et al. (2007) and purified by methanol/chloroform precipitation. An amount of $40 \mu \mathrm{g}$ of control or experimental protein samples was stained with $400 \mathrm{pmol}$ of Cy3 or Cy5 (CyDryes manufacturer, GE Healthcare). Standard control for normalization between gels consisted of a pool of the samples at the same concentration stained with Cy2. DiGE first dimension of electrophoresis was performed in an IPGphor IEF System with a linear 3-11 pH (GE Healthcare). DiGE second dimension was performed on an Ettan Dalt Six by using $12.5 \%$ SDS-PAGE at $20^{\circ} \mathrm{C}$. Gels were scanned at $100 \mu \mathrm{m}$ resolution in a Typhoon 9400 scanner (GE Healthcare). Data analysis was performed by using two or three replicates with DeCyder software v7.0, Differential In-Gel Analysis (DIA) and Biological Variation Analysis (BVA) for which, as filter, a fold change $\geq 1.2$ or $\leq-1.2$ was used with a Student $t$ test to extract significant results between replicates $(p<0.05)$.

The results show that a $200 \mathrm{~min}$ treatment in mechanical facilities did not produce any significant effect at the proteomic scale (Table 1). In fact, we were able to detect one spot significantly altered in the RPM and two in the LDC only when removing the most variable replicate, which suggests that we were only detecting highly significant variations. The effect of a high magnetic field is moderate, producing more decreased than increased spots; a clearly stronger effect is observed in the $0 g^{*}$ and $2 g^{*}$ positions, especially detected in the $2 g^{*}$ position. These effects are quite similar to the synergic effect found at the transcriptomic level with the same samples (Manzano et al., 2012), reinforcing the idea of an 


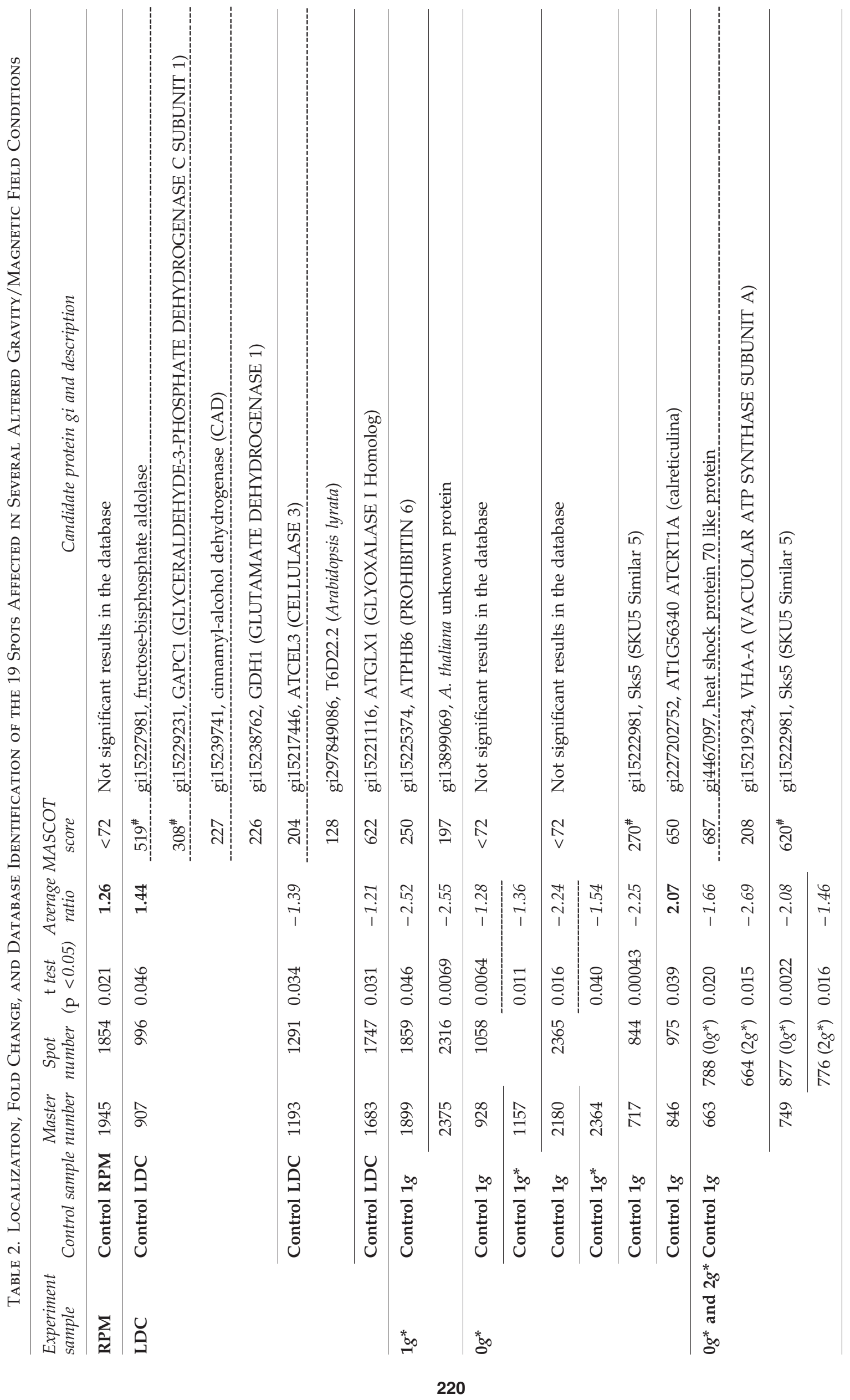




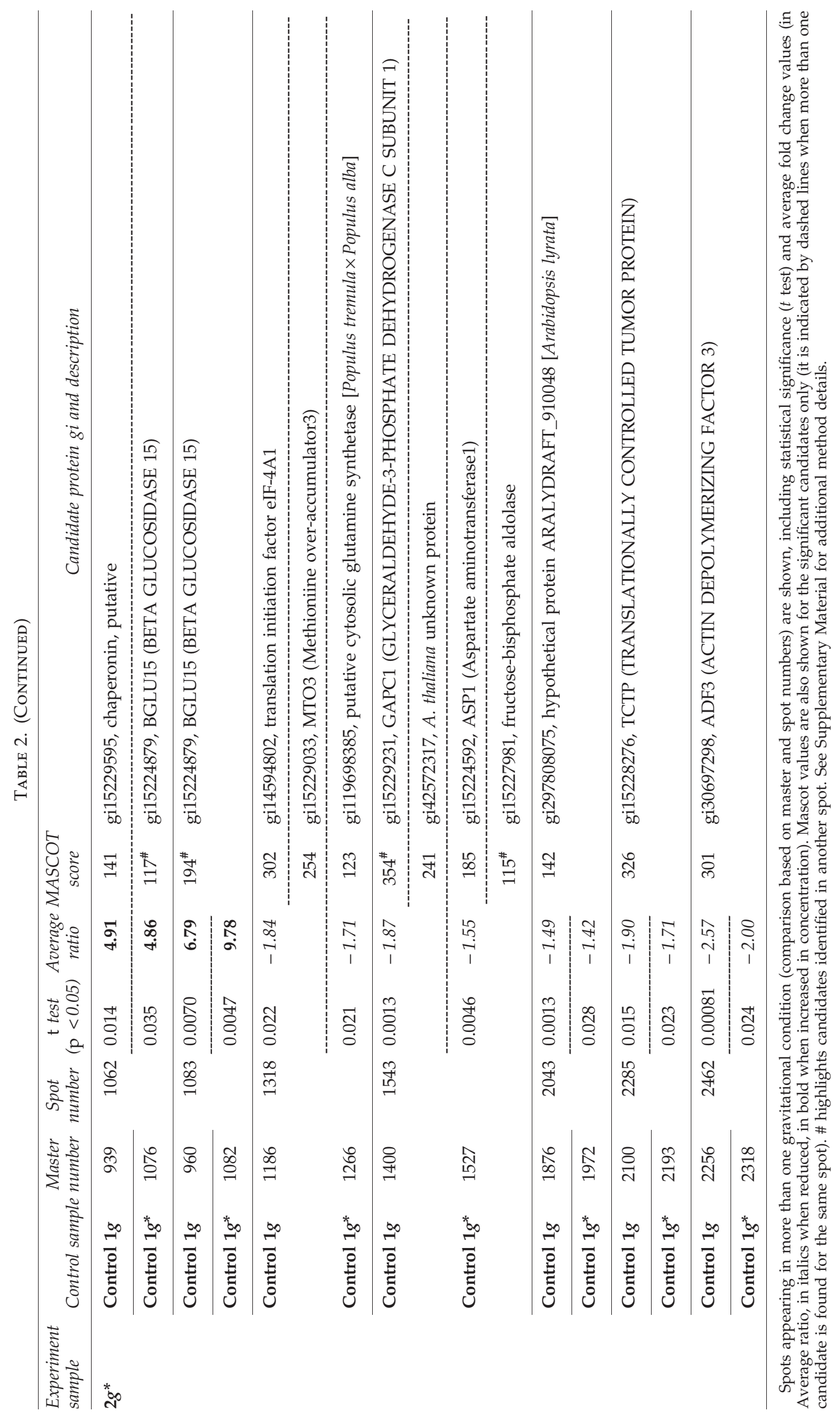


enhanced impact of altered gravity on biological systems under suboptimal environments (Herranz et al., 2010, 2012).

To analyze more deeply the proteomic response, we decided to identify the more representative altered spots in several pair comparisons. We chose 19 spots (Table 2), namely, (a) those altered in the RPM/LDC samples, (b) two spots especially easy to isolate with high fold changes in the $0 g^{*}$ position, (c) those spots appearing simultaneously altered in any $g^{*}$ level after comparing its proteomic profile with both the internal $\left(1 g^{*}\right)$ and the external $(1 g)$ controls, (d) two common spots of the comparisons of $0 g^{*}$ and $2 g^{*}$ with $1 g$, and (e) two outstanding spots revealed by the comparison of $0 g^{*}$ with the external $1 g$ control. Once the spots of interest were identified and located, trypsin-digested spots were analyzed by a 4800 MALDI-TOF-TOF facility (Maccarrone et al., 2010). MASCOT searches with nrNCBI protein database (viridiplantae only) were used to identify the candidate proteins that better fit with the mass spectrum obtained for each spot tryptic peptides, up to eight fragmentation peptides analyzed per spectrum (score higher than 72 to have a $p<0.05$ ).

Candidate proteins that appeared during the analysis were easily identified as involved in cellular response to stress conditions, like chaperonin (4.91-fold increase in $\left.2 g^{*}\right)$, $\beta$-glucosidase-15 (almost 10 -fold increase in $2 g^{*}$ ), and Hsp70 (slightly but significantly decreased in both $0 g^{*}$ and $2 g^{*}$ ), together with ATP synthase VHA-A and oxidoreductase Sks5, which appeared in two different spots. Primary and secondary metabolism enzymes usually appear to be affected by environmental stress conditions (Liu et al., 2011), and some of them were also found to be modified in our study (Table 2). Previously performed proteomic analyses with the use of 2-D electrophoresis cross comparisons identified similar types of proteins that were affected by mechanically altered gravity conditions, among which fructose-bisphosphate aldolase in particular was mentioned (Wang et al., 2006; Barjaktarovic et al., 2007, 2009). In our work, in which spots were quantified in the same gel (DiGE), only a few variations were found to be significant in the mechanical facilities, whereas the synergic action of two stressing conditions (magnetic field and altered gravity) revealed proteomic changes similar to those reported in the literature.

Finally, we compared these specific proteins with the genes we previously found to be affected with the use of an Agilent array on the same samples. Most of the identified matches affected similarly both hypergravity environments $\left(2 g^{*}\right.$ in the magnet and LDC) (Table 3). It must be kept in mind that the decrease of one spot could mean that the affected protein has been postranslationally modified (transformed into a new spot), becoming even more active; this would explain apparent inconsistencies between proteomic and transcriptomic data sets. In fact, we found additional partial matches [e.g., protein GAPC-1, used as example in Fig. 1C, match gene is not affected; but a similar gene, GAPA-2 (AT1G12900), is] that would cross-validate both proteomic and transcriptomic data sets.

Summarizing, we conclude that a $200 \mathrm{~min}$ exposure to mechanically altered gravity produces very few alterations in our environmentally controlled system in comparison with other studies in which Arabidopsis callus was exposed to clinorotation or centrifugation with other designs (Wang et al., 2006; Barjaktarovic et al., 2007, 2009). This result can be explained by the different sensitivities of other proteomic approaches (DiGE technology produces more statistically reliable results than independent 2-D gels but provides less number of spots detected) and also by the different duration of the treatment and source of the biological material (for instance, when using seedlings, cellular plus tissular proteomic responses will be observed). Nevertheless, our results are consistent with those of previous studies, which reinforces the idea that the alteration of gravity, together with other physical forces, promotes an abiotic stress response that provides the cells some adaptation benefits in the context of a new environmental situation at genomic and proteomic levels (Barjaktarovic et al., 2007, 2009; Manzano et al., 2012). In fact, several plant species have the capacity to cope with extreme environmental conditions by modifying secondary metabolism, stress, and repair pathways in a concerted way (Van Cutsem et al., 2011; Forster et al., 2012; Payyavula et al., 2012).

Using magnetic forces seems to increase the system susceptibility to altered gravity and affects similar proteins as those that have been described in other studies in which mechanical simulators were used. The same gene ontology functions have been detected by transcriptomic analyses (Manzano et al., 2012). Similar enhanced effects of microgravity on the transcriptomic profile have been observed before when suboptimal environmental conditions were added to the altered gravity stimulus (Herranz et al., 2010, 2012; Manzano et al., 2012). It is possible that the observed

Table 3. Coincidences between Proteomic and Transcriptomic Identified Protein/Gene

\begin{tabular}{|c|c|c|c|}
\hline \multirow[b]{2}{*}{ Protein description } & Proteome changes & \multirow[b]{2}{*}{ Array ID } & \multirow{2}{*}{$\begin{array}{c}\text { Transcriptome changes } \\
\text { Affected positions }\end{array}$} \\
\hline & Affected positions & & \\
\hline gi15227981, fructose-bisphosphate aldolase & LDC and $2 g^{*}$ & AT2G36460 & $L D C$ and $2 g^{*}$ \\
\hline $\begin{array}{l}\text { gi15229231, GAPC1 (Glyceraldehyde-3-phosphate } \\
\text { dehydrogenase c subunit 1) }\end{array}$ & LDC and $2 g^{*}$ & AT3G04120 & $L D C$ and $2 \mathbf{g}^{*}$ \\
\hline gi15239741, CAD (Cinnamyl-alcohol dehydrogenase) & $L D C$ & AT3G19450 & $L D C$ \\
\hline gi15238762, GDH1 (Glutamate dehydrogenase 1) & LDC & AT5G18170 & LDC and $O g^{*}$ \\
\hline gi14594802, translation initiation factor eIF-4A1 & $2 g^{*}$ & AT1G51380 & $2 g^{*}$ and LDC \\
\hline gi15224592, ASP1 (Aspartate aminotransferase 1) & $2 g^{*}$ & AT2G30970 & $2 \mathrm{~g}^{*}$ \\
\hline $\begin{array}{l}\text { gi15228276, TCTP (Translationally controlled } \\
\text { tumor protein) }\end{array}$ & $2 g^{*}$ & AT3G16640 & $2 g^{*}$ and $L D C$ \\
\hline
\end{tabular}

Changes in gene expression or protein levels are indicated in bold (increase) or italics (decrease). 
effects at the $0 g^{*}$ and $2 g^{*}$ positions could be related to forces generated by differences in magnetic susceptibility between different components of the cell. Further experiments are required to assess this possibility.

Investigation of "pure" microgravity effects should be performed in space, but mechanical and magnetic simulators could be used to study similar phenomena if we are able to distinguish the mechanical/magnetic effects from the gravitational effects in our systems. In addition, magnetic levitation can be an alternative to other ground-based methodologies and allow us to test the biological effects of altered gravitational forces in an unusual environment.

\section{Supplementary Material}

All raw and identification data is available as Supplementary Material (available online at www.liebertonline.com/ast and deposited in the PRIDE database (http://www.ebi.ac.uk/ pride) following CNB computational proteomics unit/ proteomics facility guidelines (Kenyani et al., 2011) (PRIDE ID: 22049, 22050).

\section{Acknowledgments}

The authors acknowledge funding from the Spanish Space Program in the "Plan Nacional de Investigacion Cientifica y Desarrollo Tecnologico" AYA2009-07952, AYA2010-11834-E to F.J.M. and AYA2009-07792-E to R.H. RPM/LDC access was possible due to the Dutch Space Research Organization NWO-ALW-SRON grant MG-057 to J.vL. and the SEGMGSPE_Ph1 access to ESA GBF project contract 4200022650 to R.H., F.J.M., and J.vL. Magnetic levitation at the High Field Magnet Laboratory in Nijmegen was granted by EuroMagNET II under the EU contract $n^{\circ} 228043$ and by the Stichting voor Fundamenteel Onderzoek der Materie (FOM), financially supported by the Nederlandse Organisatie voor Wetenschappelijk Onderzoek (NWO). PRIDE files have been generated in the CSIC CNB computational proteomics unit from CSIC \& ProteoRed: Carlos III Networked Proteomics Platform. We want to acknowledge also Dr. Mateos and two anonymous referees for their critical review of the manuscript.

\section{Author Disclosure Statement}

The authors have declared no conflict of interest.

\section{Abbreviations}

BVA, Biological Variation Analysis; DIA, Differential InGel Analysis; DiGE, difference gel electrophoresis; LDC, Large Diameter Centrifuge; RPM, Random Positioning Machine.

\section{References}

Barjaktarovic, Z., Nordheim, A., Lamkemeyer, T., Fladerer, C., Madlung, J., and Hampp, R. (2007) Time-course of changes in amounts of specific proteins upon exposure to hyper-g, 2-D clinorotation, and 3-D random positioning of Arabidopsis cell cultures. L Exp Bot 58:4357-4363.

Barjaktarovic, Z., Schutz, W., Madlung, J., Fladerer, C., Nordheim, A., and Hampp, R. (2009) Changes in the effective gravitational field strength affect the state of phosphorylation of stress-related proteins in callus cultures of Arabidopsis thaliana. J Exp Bot 60:779-789.

Beaugnon, E. and Tournier, R. (1991a) Levitation of organic materials. Nature 349:470.

Beaugnon, E. and Tournier, R. (1991b) Levitation of water and organic substances in high static magnetic fields. Journal de Physique III France 1:1423-1428.

Berry, M.V. and Geim, A.K. (1997) Of flying frogs and levitrons. European Journal of Physics 18:307-313.

Beuls, E., Van Houdt, R., Leys, N., Dijkstra, C., Larkin, O., and Mahillon, J. (2009) Bacillus thuringiensis conjugation in simulated microgravity. Astrobiology 9:797-805.

Dijkstra, C.E., Larkin, O.J., Anthony, P., Davey, M.R., Eaves, L., Rees, C.E., and Hill, R.J. (2011) Diamagnetic levitation enhances growth of liquid bacterial cultures by increasing oxygen availability. $L R$ Soc Interface 8:334-344.

Forster, F., Beisser, D., Grohme, M.A., Liang, C., Mali, B., Siegl, A.M., Engelmann, J.C., Shkumatov, A.V., Schokraie, E., Muller, T., Schnolzer, M., Schill, R.O., Frohme, M., and Dandekar, T. (2012) Transcriptome analysis in tardigrade species reveals specific molecular pathways for stress adaptations. Bioinform Biol Insights 6:69-96.

Guevorkian, K. and Valles, J.M., Jr. (2006) Swimming Paramecium in magnetically simulated enhanced, reduced, and inverted gravity environments. Proc Natl Acad Sci USA 103:13051-13056.

Hammer, B.E., Kidder, L.S., Williams, P.C., and Xu, W.W. (2009) Magnetic levitation of MC3T3 osteoblast cells as a groundbased simulation of microgravity. Microgravity Sci Technol 21:311-318.

Herranz, R., Benguria, A., Lavan, D.A., Lopez-Vidriero, I., Gasset, G., Medina, F.J., van Loon, J.J., and Marco, R. (2010) Spaceflightrelated suboptimal conditions can accentuate the altered gravity response of Drosophila transcriptome. Mol Ecol 19:4255-4264.

Herranz, R., Larkin, O.J., Dijkstra, C.E., Hill, R.J.A., Anthony, P., Davey, M.R., Eaves, L., van Loon, J.J.W.A., Medina, F.J., and Marco, R. (2012) Microgravity simulation by diamagnetic levitation: effects of a strong gradient magnetic field on the transcriptional profile of Drosophila melanogaster. BMC Genomics 13:52.

Herranz, R., Anken, R., Boonstra, J., Braun, M., Christianen, P.C.M., de Geest, M., Hauslage, J., Hilbig, R., Hill, R.J.A., Lebert, M., Medina, F.J., Vagt, N., Ullrich, O., van Loon, J.J.W.A., and Hemmersbach, R. (2013) Ground-based facilities for simulation of microgravity, including terminology and organism-specific recommendations for their use. Astrobiology 13:1-17.

Hill, R.J., Larkin, O.J., Dijkstra, C.E., Manzano, A.I., de Juan, E., Davey, M.R., Anthony, P., Eaves, L., Medina, F.J., Marco, R., and Herranz, R. (2012) Effect of magnetically simulated zerogravity and enhanced gravity on the walk of the common fruitfly. $L R$ Soc Interface 9:1438-1449.

Hoson, T., Kamisaka, S., Masuda, Y., and Yamashita, M. (1992) Changes in plant growth processes under microgravity conditions simulated by a three-dimensional clinostat. The Botanical Magazine 105:53-70.

Kenyani, J., Medina-Aunon, J.A., Martinez-Bartolome, S., Albar, J.P., Wastling, J.M., and Jones, A.R. (2011) A DIGE study on the effects of salbutamol on the rat muscle proteome-an exemplar of best practice for data sharing in proteomics. $\underline{B M C}$ Res Notes 4:86.

Kraft, T.F., van Loon, J.J., and Kiss, J.Z. (2000) Plastid position in Arabidopsis columella cells is similar in microgravity and on a random-positioning machine. Planta 211:415-422. 
Liu, M., Gao, H., Shang, P., Zhou, X., Ashforth, E., Zhuo, Y., Chen, D., Ren, B., Liu, Z., and Zhang, L. (2011) Magnetic field is the dominant factor to induce the response of Streptomyces avermitilis in altered gravity simulated by diamagnetic levitation. PLoS One 6, e24697.

Maccarrone, G., Turck, C.W., and Martins-de-Souza, D. (2010) Shotgun mass spectrometry workflow combining IEF and LCMALDI-TOF/TOF. Protein I 29:99-102.

Manzano, A.I., van Loon, J.J.W.A., Christianen, P., GonzalezRubio, J.M., Medina, F.J., and Herranz, R. (2012) Gravitational and magnetic field variations synergize to reveal subtle variations in the global transcriptional state of Arabidopsis in vitro callus cultures. BMC Genomics 13:105.

Menges, M. and Murray, J.A. (2006) Synchronization, transformation, and cryopreservation of suspension-cultured cells. Methods Mol Biol 323:45-61.

Payyavula, R.S., Navarre, D.A., Kuhl, J.C., Pantoja, A., and Pillai, S.S. (2012) Differential effects of environment on potato phenylpropanoid and carotenoid expression. BMC Plant Biol 12:39.

Perenboom, J.A.A.J., Wiegers, S.A.J., Christianen, P.C.M., Zeitler, U., and Maan, J.C. (2004) The new installation at the Nijmegen High Field Magnet Laboratory. Physica B Condens Matter 346347:659-662.

Schenck, J.F. (1992) Health and physiological effects of human exposure to whole-body four-tesla magnetic fields during MRI. Ann NY Acad Sci 649:285-301.

Valles, J.M., Jr., Lin, K., Denegre, J.M., and Mowry, K.L. (1997) Stable magnetic field gradient levitation of Xenopus laevis: toward low-gravity simulation. Biophys I 73:1130-1133.

Valles, J.M., Jr., Maris, H.J., Seidel, G.M., Tang, J., and Yao, W. (2005) Magnetic levitation-based martian and lunar gravity simulator. Adv Space Res 36:114-118.

Van Cutsem, E., Simonart, G., Degand, H., Faber, A.M., Morsomme, P., and Boutry, M. (2011) Gel-based and gel-free proteomic analysis of Nicotiana tabacum trichomes identifies proteins involved in secondary metabolism and in the (a)biotic stress response. Proteomics 11:440-454.

van Loon, J.J., Folgering, E.H., Bouten, C.V., and Smit, T.H. (2004) Centrifuges and inertial shear forces. I Gravit Physiol 11:29-38. van Loon, J.J.W.A. (2007) Some history and use of the Random Positioning Machine, RPM, in gravity related research. $\underline{A d v}$ Space Res 39:1161-1165.

van Loon, J.J.W.A., Krause, J., Cunha, H., Goncalves, J., Almeida, H., and Schiller, P. (2008) The large diameter centrifuge, LDC, for life and physical sciences and technology. In Proceedings of the Symposium "Life in Space for Life on Earth," ESA SP-663, edited by L. Ouwehand, European Space Agency, ESTEC, Noordwijk, the Netherlands. Abstract P1-005.

Wang, H., Zheng, H.Q., Sha, W., Zeng, R., and Xia, Q.C. (2006) A proteomic approach to analysing responses of Arabidopsis thaliana callus cells to clinostat rotation. L Exp Bot 57:827-835.

Wiegers, S.A.J., Christianen, P.C.M., Engelkamp, H., den Ouden, A., Perenboom, J.A.A.J., Zeitler, U., and Maan, J.C. (2010) The High Field Magnet Laboratory at Radboud University Nijmegen. LLw Temp Phys 159:389-393.

Address correspondence to: Dr. Raúl Herranz Centro de Investigaciones Biológicas (CSIC) C/ Ramiro de Maeztu 9 E-28040 Madrid Spain

E-mail: r.herranz@csic.es

Dr. F. Javier Medina Centro de Investigaciones Biológicas (CSIC) C/ Ramiro de Maeztu 9 E-28040 Madrid Spain

E-mail: fjmedina@cib.csic.es

Submitted 6 June 2012 Accepted 27 November 2012 
This article has been cited by:

1. Mariano Bizzarri, Alessandra Cucina, Alessandro Palombo, Maria Grazia Masiello. 2014. Gravity sensing by cells: mechanisms and theoretical grounds. Rendiconti Lincei 25:S1, 29-38. [CrossRef]

2. Raúl Herranz, Miguel A Valbuena, Khaled Youssef, Francisco-Javier Medina. 2014. Mechanisms of disruption of meristematic competence by microgravity in Arabidopsis seedlings. Plant Signaling \& Bebavior 9:2, e28289. [CrossRef]

3. Hai-fen Li, Xiao-Ping Chen, Fang-he Zhu, Hai-Yan Liu, Yan-Bin Hong, Xuan-Qiang Liang. 2013. Transcriptome profiling of peanut (Arachis hypogaea) gynophores in gravitropic response. Functional Plant Biology 40:12, 1249. [CrossRef] 